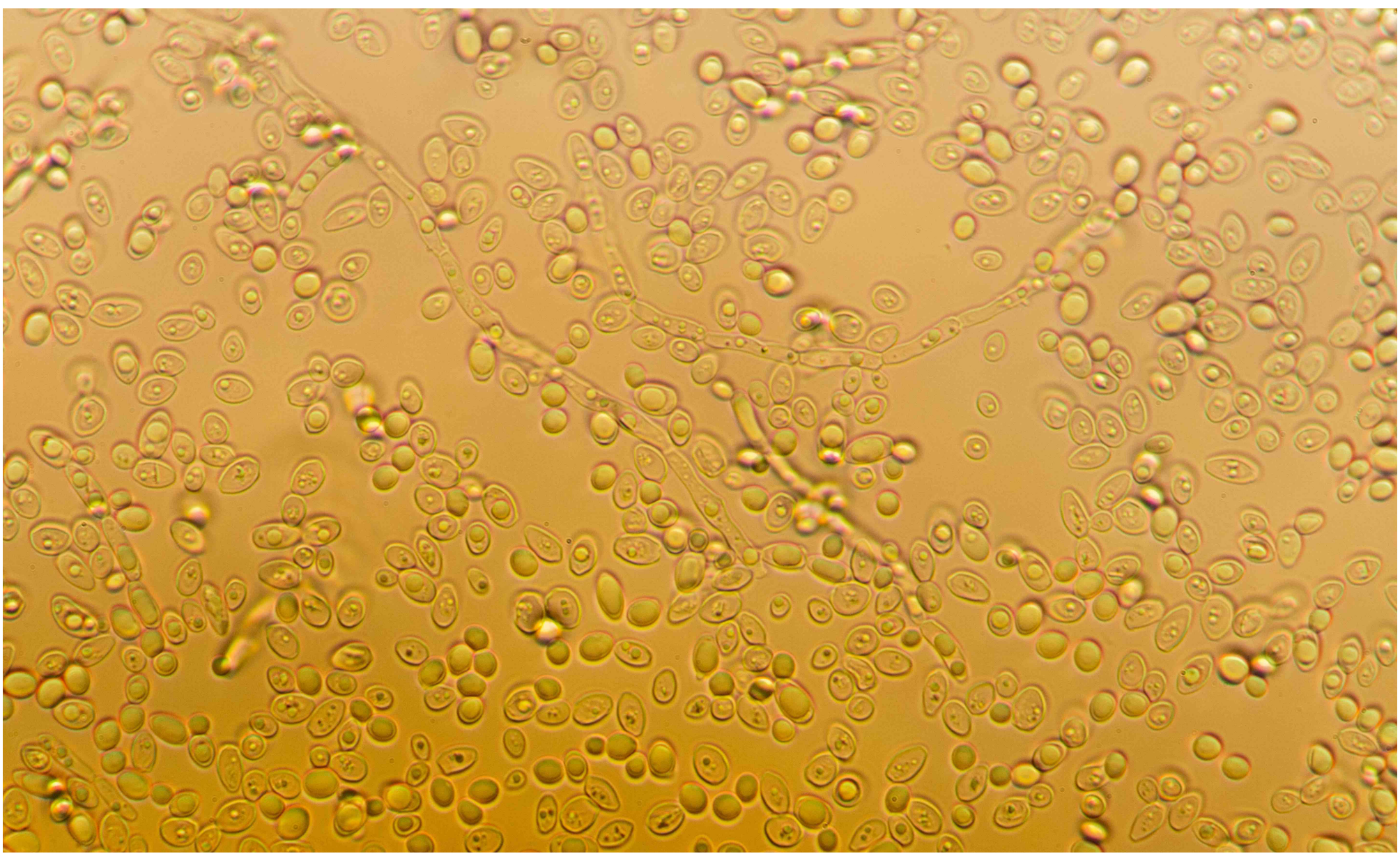

Commentary

\title{
2016 Nobel Prize Winner Yoshinori Ohsumi; Solving the Mystery of Autophagy
}

Andrei Dan

Published online: 02 January 2017

${ }^{1}$ Faculty of Medicine, McGill University, Montréal, Canada.

Corresponding Author: Andrei Dan

In 2016, Dr. Yoshinori Ohsumi accomplished an incredible feat: he received the Nobel Prize in medicine or physiology as a single recipient. Awarded once a year to up to 3 individuals who made a significant contribution to the scientific community, this Nobel Prize is the most well known, and arguably the most prestigious prize for those studying the human body. This year, it was Dr. Ohsumi alone who was given the honour for his groundbreaking work on autophagy. 
Born in 1945 in Fukuoka, Dr. Ohsumi grew up in difficult times. He survived a tough childhood in a country recovering from World War 2 and his mother was severely ill from tuberculosis. He sought solace in nature, observing the stars and collecting insects. These experiences lead him to pursue his studies at University of Tokyo, followed by graduate studies at the same school working on ribosomes and protein synthesis. His graduate work culminated to his post-doctoral fellowship at the Rockefeller University, experimenting with DNA replication in yeast.

It was 1963, while Dr. Ohsumi was still in his undergraduate studies, that Christian de Duve coined the term autophagy, and extensively discussed this concept in a review article published a few years later. Relatively unknown to many at the time, autophagy, is the process through which the cell recycles its materials. Autophagy is essential to all processes in the organism, from embryogenesis to daily function. It is conserved from unicellular yeast to complex mammals. It was speculated that when a cell is under stress, it breaks down large proteins to reuse the metabolites as building blocks.

Similarly, when a cell needs less energy, it no longer needs as many mitochondria and autophagy is triggered to break down excessive mitochondria. It was known that cells could undergo such a process. However, the mechanism of autophagy was unclear.

In 1977, after his fellowship at the Rockefeller University, Dr. Ohsumi was hired as an assistant professor at the University of Tokyo where he first postulated what would be his most brilliant idea. He hypothesized that if autophagy was truly an important pathway, it would have to be conserved throughout evolution. Thus, the yeast, which he was working with, would need a way to undergo autophagy. In fact, a yeast has a vacuole, which is the functional equivalent of the mammalian lysosome, the organelle that is most likely to be involved in autophagy since it contains powerful digestive enzymes. Ohsumi reasoned that if autophagy existed in the yeast, inhibition of vacuolar digestive enzymes would result in the accumulation of engulfed cytoplasmic components in the vacuole, and he was right.

He proceeds to consider the following scenario: something must move these cellular components in order to start the autophagy process. By inducing random mutations in yeast cells lacking vacuolar proteases, Ohsumi identified the first mutant that could not accumulate autophagic bodies in the vacuole: gene autophagy 1 (APG1). He continues to identify the genes APG1-15, mapping out the key pathway, including the creation of the autophagosome, the specific structure responsible for autophagy in humans. As a result, he opened the door for hundreds of other scientists to investigate this pathway and explore what kind of effects it would have on a multicellular, complex organism.

Decades later, autophagy has evolved to a topic so diverse that it has gained widespread application in clinical medicine. The field has grown from 20 publications a year in the 1970s to over 5000 annual publications today, many of which are translational and clinical research aimed at treating many of the most challenging medical conditions. For instance, BECN1 is part of the autophagy cycle and has been implicated in breast and ovarian cancer development. Tissue-specific disruption of the other two genes involved in autophagy, Atg5 and Atg7, causes neurodegeneration in mouse models. Autophagy even has hypothesized cytoprotective functions and the capacity to counteract cell injury and diseases associated with aging.

The ongoing research is aimed at developing new understanding, new therapies and new hope for thousands of patients, all because of the pioneering work of Dr. Ohsumi, the man who mapped out the pathway of autophagy. 


\section{References}

"Yoshinori Ohsumi - Nobel Lecture". Nobelprize.org. Nobel Media AB 2014. Web. 18 Dec 2016.

Nils-Göran Larsson and Maria G. Masucci Scientific Background for the 2016 Nobel Prize in Physiology or Medicine. Karolinska Institutet Web. 18 Dec 2016.

\section{(9) $(0 \otimes$}

This work is licensed under a Creative Commons Attribution-NonCommercialShareAlike 4.0 International License

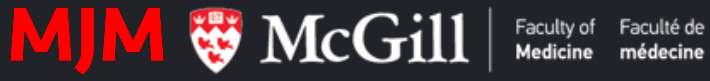

(c) McGill Journal of Medicine 2020

Electronic ISSN 1715-8125

\section{ffy}

